\title{
Modelling and Simulation of Stepper Motor For Position Control Using LabVIEW
}

\author{
Ahmet Mehmet KARADENIZ \\ Department of Mechatronics \\ University of Debrecen \\ Debrecen, Hungary \\ ahmetm.karadeniz@gmail.com
}

\author{
Malek ALKAYYALI \\ Department of Mechatronics \\ University of Debrecen \\ Debrecen, Hungary \\ malekkayyali@gmail.com
}

\author{
Dr. Peter Tamas SZEMES \\ Department of Mechatronics \\ University of Debrecen \\ Debrecen, Hungary \\ szemespeter@eng.unideb.hu
}

\begin{abstract}
This paper presents hybrid stepper motor (is a type of stepping motor) modelling and simulation which is widely used a kind of motor in industrial applications. In this study, the stepper motor was modelled using bond graph technique and simulation for desired position was executed on LabVIEW graphical interface. Then, firstly a convenient PID controller was designed for position, speed and current and PID close loop response was obtained for position control. Then, PID parameters for each controller were arranged separately to obtain good response Secondly, Fuzzy Logic controller applied to the system and its response was obtained. Finally, both responses are compared. According to comparison, it was observed that Fuzzy Logic controller's response is better than PID's. (In this paper, all shown responses were observed for 120 degree desired position)
\end{abstract}

Keywords-stepper motor, position control, bond graph modelling, PID controller, Fuzzy Logic.

\section{INTRODUCTION}

Stepper Motors can be defined as DC Motors that can move in discrete steps. They have multiple coils. Each phase is energized in order to provide step by step rotation of motor. Then, highly precise positioning and speed control can be obtained in computer controlled stepping applications. Therefore, stepper motors are the most preferred motors for many precise motion control applications.

There are three type of stepper motors: permanent magnet stepper motors, variable reluctance stepper motors (it may named switched reluctance stepper motors) and hybrid stepper motors [1]. In this paper, the study for hybrid stepper motor application was performed.

Hybrid Stepper Motors are widely used in highly precise position controlled applications such as robotic systems, 3d printers, etc. [2]. This type of stepper motors have all features that other types of stepper motors behave. Hybrid stepper motors use disc rotor which is magnetized longitudinal to produce small step angle and low inertia torque. Therefore, hybrid stepper motors are more costly via according to other type of stepper motors and have more complex structure [3].

The rest of this paper is organized as follows. The stepper motor and its mathematical model is introduced and the bond graph technique is explained and stepper motor mathematical model and bond graph modelling is described in in Section II. Section III explains the design of the general PID controller and introduces the close loop position control. Finally, the conclusions are summarized in Section IV.

\section{STEPPER MOTOR MODELING}

\section{A. Mathematical Modelling}

In this section, the mathematical model of a hybrid stepper motor is derived. Fig. 1 shows the schematic drawing of a simple two-phase hybrid stepper motor with three teeth [4].

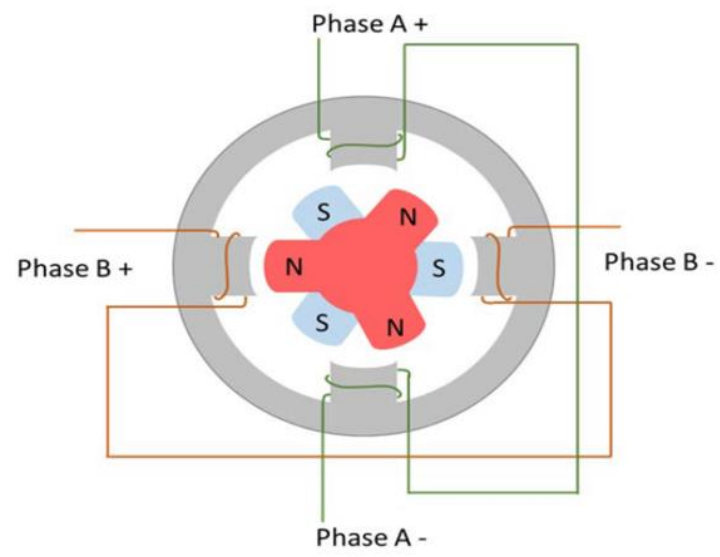

Fig. 1: Schematic view of a stepper motor with three teeth[4].

The mathematical model of a stepper model could be divided into two sub-models, electrical model and mechanical model. Starting with the electrical model, each phase of the stepper motor phases could be modelled as an RL circuit plus a back electromotive force (emf) in Figure 2[4]. According to [4] the differential equations of phase $\mathrm{a}$ and phase $\mathrm{b}$ are given by (1) and (2), respectively,

$$
\begin{aligned}
& L_{a} \frac{d i_{a}(t)}{d t}=-R_{a} i_{a}(t)-e_{a}(t)+u_{a}(t) \\
& L_{b} \frac{d i_{b}(t)}{d t}=-R_{b} i_{b}(t)-e_{b}(t)+u_{b}(t)
\end{aligned}
$$


where: $\quad e_{a}(t)=K_{m} \omega_{m} \sin \left(p \theta_{m}\right) \quad$ and $\quad e_{b}(t)=$ $K_{m} \omega_{m} \cos \left(p \theta_{m}\right)$.

In equation (1) and (2), the phase resistances and inductances are assumed to be equal, $R_{a}=R_{b}=R[\Omega]$ and $L_{a}=L_{b}=L[\mathrm{H}]$. Moreover, $u_{a}(t)$ and $u_{b}(t)$ are the terminal voltages [V], $e_{a}(t)$ and $e_{b}(t)$ are the back emf [V], $K_{m}$ is the motor constant, $p$ is the number or motor poles pairs (teeth), $\omega_{m}$ is the rotor (mechanical) angular speed $[\mathrm{rad} / \mathrm{s}]$, and $\theta_{m}$ is the rotor (mechanical) angular position [rad].
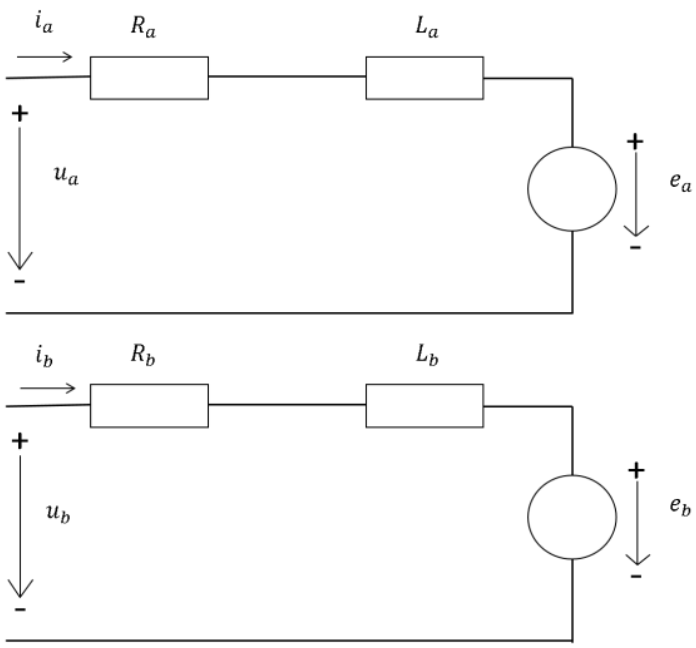

Fig. 2: The equivalent circuits of hybrid stepper motor.

The shaft of the hybrid stepper motor, which represents the mechanical part of the system, is modelled as a rigid body subjected to different torques as shown in Fig. 3 [4]. The differential equation of the mechanical sub-system is given by (3),

$$
J_{m} \frac{d \omega_{m}}{d t}=\tau_{e m}-B \omega_{m}-\tau_{d m}-\tau_{l}
$$

where: $\quad \tau_{e m}=K_{m}\left(-i_{a} \sin \left(p \theta_{m}\right)+i_{b} \cos \left(p \theta_{m}\right)\right)$ and $\tau_{d m}=T_{d m} \sin \left(2 p \theta_{m}+\alpha\right)$.

In the mechanical model, $J_{m}\left[\mathrm{~kg} \cdot \mathrm{m}^{2}\right]$ is the motor moment of inertia, $B[\mathrm{~kg} / \mathrm{s.m}]$ is the motor viscous friction coefficient, $\tau_{e m}[N . m]$ is the electromagnetic torque, $T_{d m}$ $[N . m]$ is the detent torque applied, $\alpha$ is the phase shift related to $\tau_{d m}$, and $\tau_{l}[N . m]$ is the applied load torque.

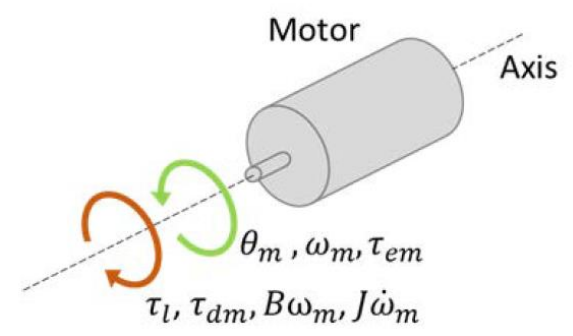

Fig. 3: The mechanical sub-system of the hybrid stepper motor[4].

\section{B. Bond Graph Modelling}

Bond Graphs are a graphical approach for diagramming the distribution and flow of power and energy within a dynamic system. More specifically, bond graphing is a unified graphical approach for modelling the storage, dissipation, and transformation of energy within a dynamic system. The structure of the bond graph has been designed to facilitate systematic derivation of differential equations governing the dynamic response of the system model. The bond graph approach leads directly to a computer simulation of the dynamic response because it delineates the causal relationships between elements or components of the model. That is, the bond graph accounts for the input/output relations between elements and subsystems of the model[5][7].

According to stepper motor model in Fig. 2, the bond graph

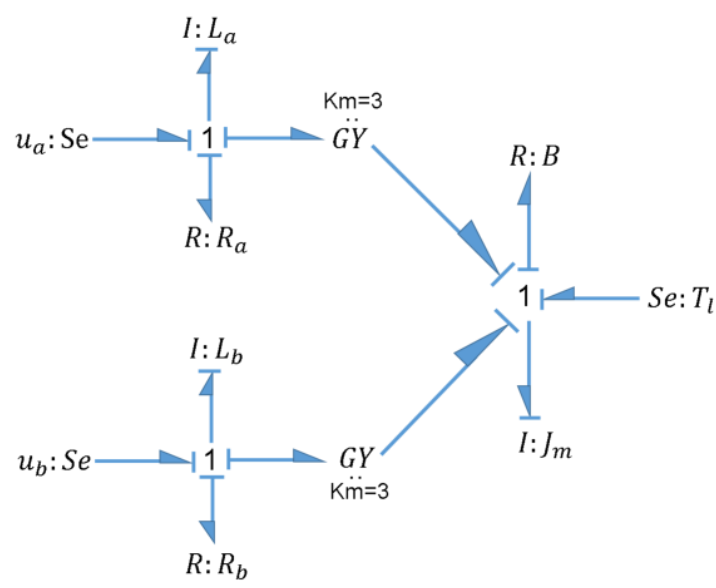

Fig. 4: Bond graph modelling of hybrid stepper motor.

model of hybrid stepper motor is shown in Fig. 4.

\section{Closed LoOp Position CONTROL, PID AND FuZZY LoGIC CONTROLLERS OF HYBRID STEPPER MOTOR}

The behaviour of the system as observed by a signal transducer or sensor is compared with the desired or command value of that variable, and the difference or error is sent to a controller, which in turn directs the actuator such that it reduces the error. In complex systems the actuators themselves are imbedded control subsystems, with their own brains, sensors and muscle.

In order to control the position of hybrid stepper motor, field oriented control (FOC) method is used in this research. The main idea of FOC is to change the electrical signals from one coordinate to another. This transformation is done to transform these signals from the reference frame linked to stator phases to the rotating frame linked to the rotor to control the produced torque precisely since it is directly related to the current in one of the axis of this new frame [4].

FOC is the most commonly used method to control AC motors especially induction motors. It is used to control the 
speed and position of the motor by controlling its torque, this controlling technique achieves a very precise response close to DC motors drives. The main advantages of FOC are the very fast transient response and smooth operation. Moreover, according to the literature, this controlling method is used with synchronous machines and stepper motors [4].

According to [4], park transformation is used to transform the electrical model from $a$ and $b$ frame to $d$ and $q$ frame. The new transformed electrical model is given by (4) and (5).

$$
\begin{gathered}
L \frac{d i_{d}}{d t}=-R i_{d}+p L i_{q} \omega_{m}+u_{d} \\
L \frac{d i_{q}}{d t}=-R i_{q}-p L i_{d} \omega_{m}+u_{q}-K_{m} \omega_{m}
\end{gathered}
$$

As can be seen from (4) and (5) the currents $i_{d}$ and $i_{q}$ are coupled, [4] proposed a method to decouple them, the new electrical decoupled and linear system is given by (6) and (7).

$$
\begin{aligned}
& L \frac{d i_{d}}{d t}=-R i_{d}+u_{d}^{l i n} \\
& L \frac{d i_{q}}{d t}=-R i_{q}+u_{q}^{l i n}
\end{aligned}
$$

In order to achieve this decoupling, the electrical drive system adds the quantities $u_{d}^{\text {dec }}$ and $u_{q}^{\text {dec }}$ to the desired $u_{d}^{\text {lin }}$ and $u_{q}^{\text {lin }}$. The equations of these two quantities are given by
(8) and (9).

$$
\begin{gathered}
u_{d}^{d e c}=-L p \omega_{m} i_{q} \\
u_{q}^{d e c}=L p \omega_{m} i_{d}+K_{m} \omega_{m}
\end{gathered}
$$

The block diagram of the decoupled system with the electrical drive subsystem, electrical subsystem in $\mathrm{d}$ and $\mathrm{q}$ frame, and the mechanical subsystem is shown in Fig. 5 [4].

Now, the configuration of the motor closed-loop drive system mentioned in [6] is considered in this research. In this configuration, three controllers are required, one for the position, the other for the speed and the third one for the current. The most inner loop is the current controller loop and it has the largest bandwidth. The most outer loop is the position control loop. Fig. 6 shows the block diagram of this control configuration.

Fuzzy theory was firstly introduced by Lotfi A. Zadeh in the middle of 1960s. Nowadays, many industrial products use fuzzy controllers to control its operation since it is an automated mechanism that depends on linguistic rules to achieve a desired output or response. Fuzzy logic controllers are in general nonlinear and there is no simple mathematical equation that can describe them. But it is widely used in control applications because of the following reason: its simplicity since it uses if-then structured rules which allows

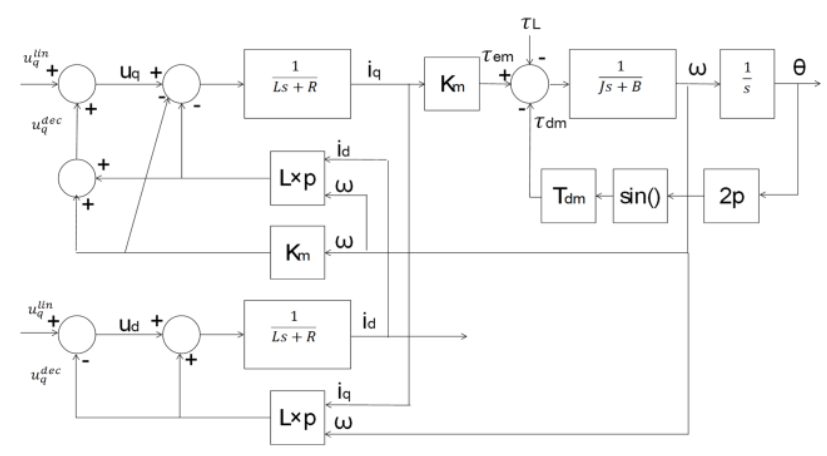

Fig. 5: Block diagram of decoupled stepper motor.

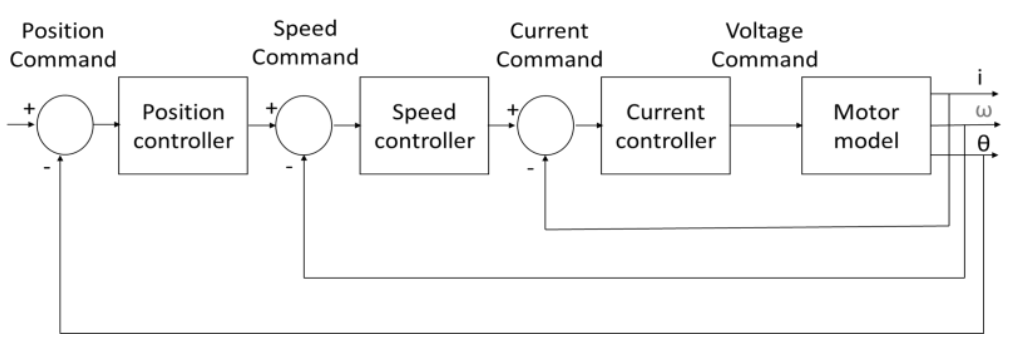

Fig. 6: Closed loop control system of stepper motor. 
the designer to take them from the expert and embed them in the controller.[9].

\section{LABVIEW MODEL}

For the hybrid stepper motor studied in this research, the model block diagram which has the mechanical subsystem, d-phase electrical subsystem and q-phase electrical subsystem and four control loops; position loop, speed loop with four controllers is shown in Fig. 8. Since four PID and Fuzzy Logic controllers are required to control this system, subsystems for these controllers are built. The block diagram with Fuzzy Logic can be obtained only using Fuzzy Logic controller instead of PID controller. TABLE I shows the rules for Fuzzy Logic controller, Fig. 7 shows the structure of PID controller and Fig. 9 shows the structure of Fuzzy Logic controller inside LabVIEW block diagram. Then, Fig. 10, Fig. 11 and Fig. 12 shows Fuzzy Logic membership functions ' $u$ ', 'du' and 'output' respectively.

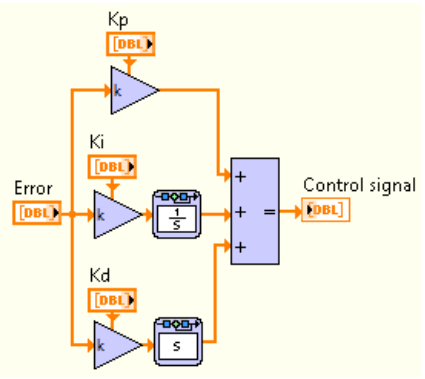

Fig. 7: LabVIEW block diagram for the PID controller.

TABLE I.

\begin{tabular}{|c|c|c|c|c|c|}
\hline \multirow{2}{*}{ du } & \multicolumn{5}{|c|}{$\mathbf{u}$} \\
\cline { 2 - 6 } & NB & NS & $Z$ & PS & PB \\
\hline NB & NB & NB & NB & NS & Z \\
\hline$Z$ & NB & NS & $Z$ & PS & PB \\
\hline PB & $Z$ & PS & PB & PB & PB \\
\hline
\end{tabular}

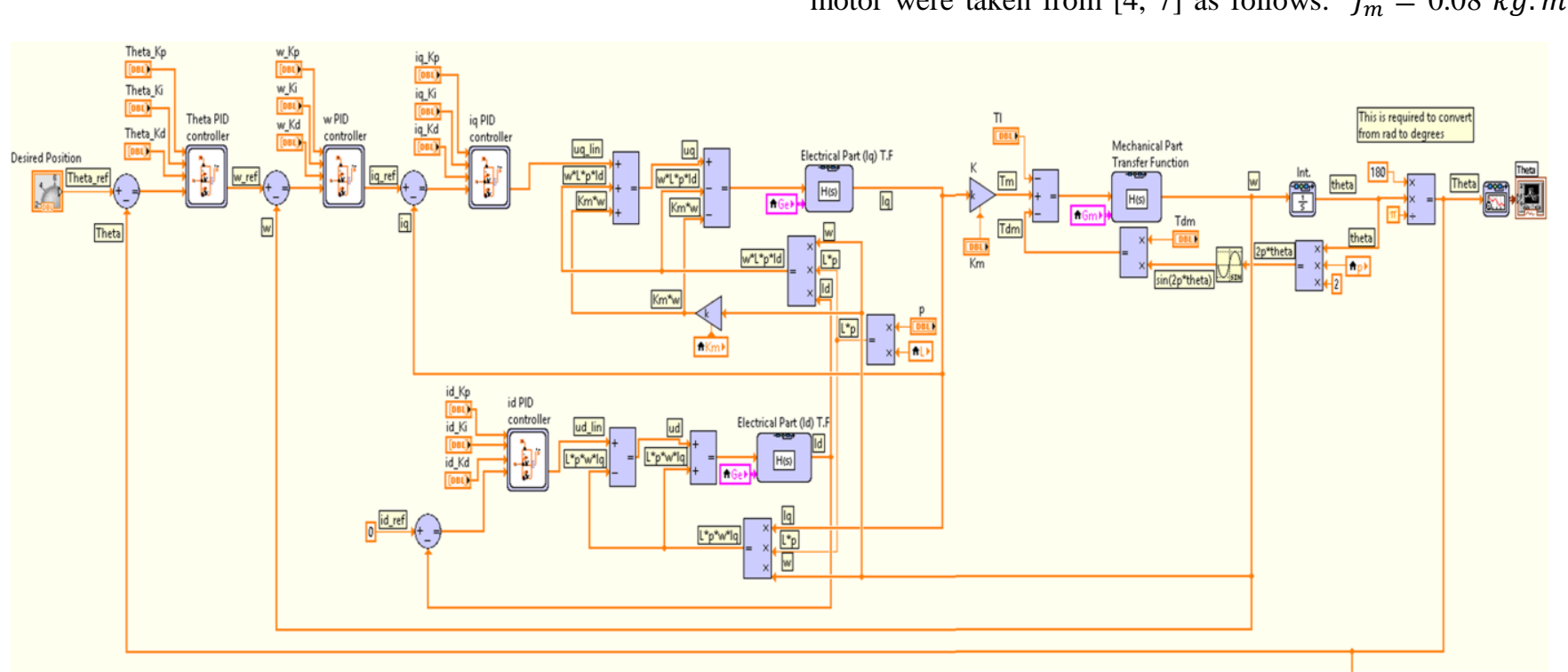

Fig. 8: LabVIEW block diagram with PID controller subsystem for the stepper motor model.

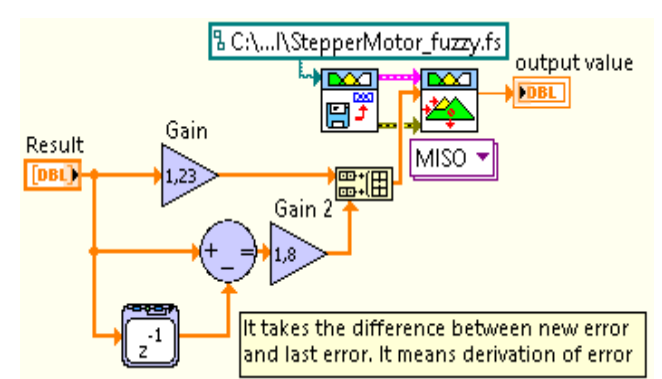

Fig. 9: LabVIEW block diagram for the Fuzzy Logic controller.

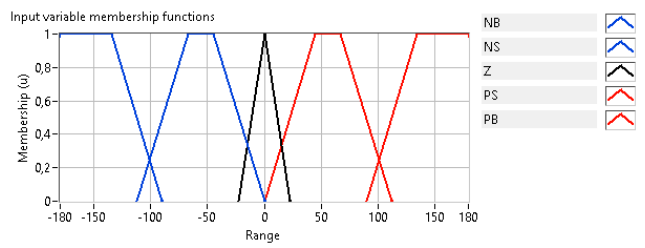

Fig. 10: Fuzzy System Designer input membership function 'u'.

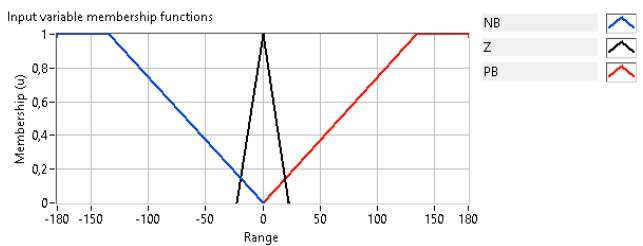

Fig. 11: Fuzzy System Designer input membership function 'du'.

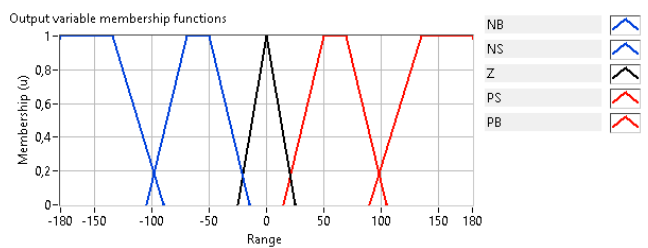

Fig. 12: Fuzzy System Designer output membership function.

\section{Simulation Results}

In this section, the simulation results are presented for the hybrid stepper motor. The parameters of the hybrid stepper motor were taken from $[4,7]$ as follows: $J_{m}=0.08 \mathrm{~kg} \cdot \mathrm{m}^{2}$, 
$B=3 \mathrm{~kg} / \mathrm{s} . \mathrm{m}, R=3 \Omega, L=3 \mathrm{H}, K_{m}=3, T_{d m}=6 \mathrm{~N} . \mathrm{m}$, $\tau_{l}=0$ N.m, $R=6$.

To make the comparison more convenient, the PID and Fuzzy Logic closed loop responses are studied. Fig. 13 shows the PID closed loop response with 120 degree desired position with tuned parameters as follows: the position control loop; $K_{p}=11, K_{i}=18.1, K_{d}=1.5 ;$ the speed control loop; $K_{p}=$ $1, K_{i}=7, K_{d}=0$; the d-phase current loop; $K_{p}=5, K_{i}=2.1$, $K_{d}=0$; the q-phase current loop; $K_{p}=6, K_{i}=17.1, K_{d}=$ 0.01. And also Fuzzy Logic controller's response is shown in Fig. 14. Furthermore, for the comparison of both controllers two responses are shown in Fig. 15.

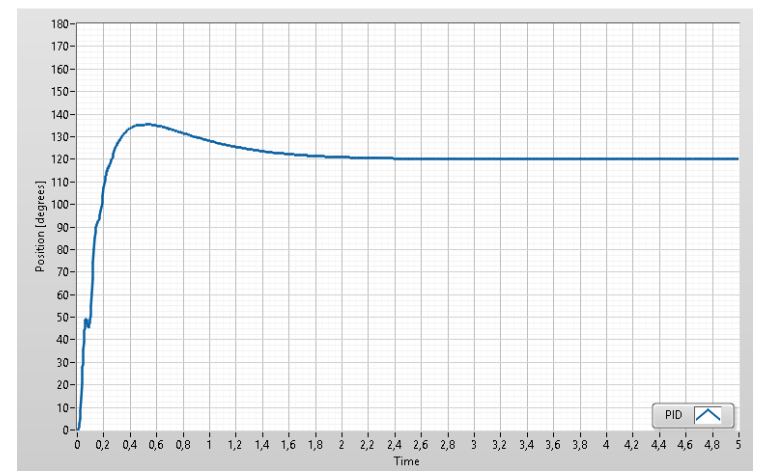

Fig. 13: PID controlled closed loop response.

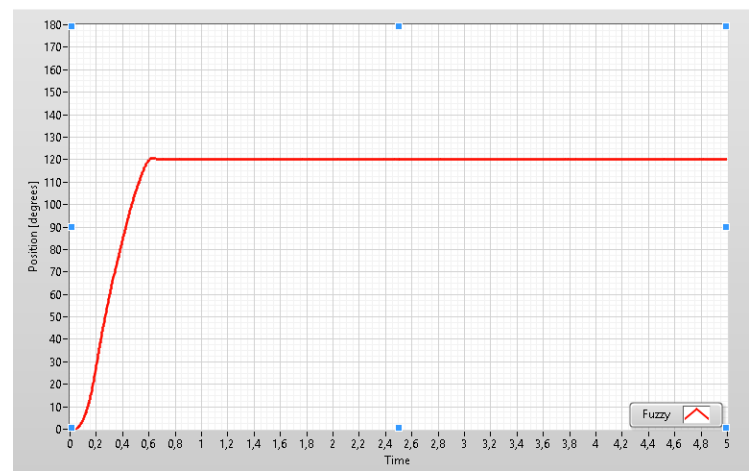

Fig. 14: Fuzzy Logic controlled closed loop response.

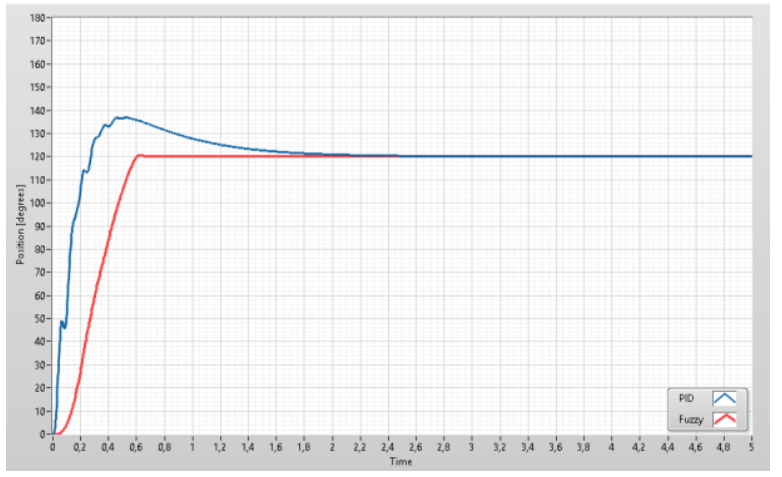

Fig. 15: Comparison of both responses.

\section{CONCLUSIONS}

One of the most efficient techniques to control the stepper motors which is field oriented control (FOC) is used to achieve the desired position. The hybrid stepper motor was studied in two modes: PID controlled closed loop and Fuzzy Logic controlled closed loop. As we noticed from the results, the motor achieves position accurately with a small overshoot using PID controller. Comparison of both controller's response on the system Fuzzy Logic's is better than PID's according to settling time and overshoot.

\section{Acknowledgment}

We acknowledge the support and help of Dr. Peter Tamas Szemes who encouraged us to work on this topic and we would like to thank our friend Ammar Alsabbagh for his support about fuzzy logic controller applications.

\section{References}

[1] A. Morar, "Stepper motor model for dynamic simulation", University of Targue-Mures, vol. 44, no. 2, pp. 117- 122, June 2003.

[2] K. M. Le, H. Van Hoang and J. W. Jeon, "An Advanced ClosedLoop Control to Improve the Performance of Hybrid Stepper Motors", IEEE Transactions on Power Electronics, vol. 32, no. 9, pp. 7244-7255, Sep. 2017

[3] M. Aydin, "Stepper Motors", Lecture Notes, Kocaeli University Mechatronics Engineering Department, May 2017.

[4] Ruilope R. P., "Modelling and Control of Stepper Motors For High Accuracy Positioning System Used in Radioactive Environments", Thesis, Politechnical University of Madrid, 2014.

[5] J. Kypuros, System Dynamics and Control with Bond Graph Modeling. CRC Press, 2013.

[6] S. H. Kim, "Ch. 2 Control of Direct Current Motors", Electric Motor Control, pp. 39-93, Elsevier, 2017.

[7] W. Borutzky, Ed., Bond Graph Modelling of Engineering Systems. New York, NY: Springer New York, 2011.

[8] N. E. I. S. U. Manual, "NI Educational Laboratory Virtual Instrumentation Suite II Series (NI ELVISTM II Series) User Manual.",2009.

[9] J. Jantzen, Foundations of fuzzy control. Chichester, England; Hoboken, NJ: John Wiley \& Sons, 2007. 